ARTICLE

DOI: $10.1038 / \mathrm{s} 41467-018-05141-4$

OPEN

\title{
A coronene-based semiconducting two- dimensional metal-organic framework with ferromagnetic behavior
}

\author{
Renhao Dong1, Zhitao Zhang ${ }^{2}$, Diana C. Tranca1', Shengqiang Zhou², Mingchao Wang ${ }^{1}$, Peter Adler ${ }^{3}$, \\ Zhongquan Liao ${ }^{4}$, Feng Liu (1) ${ }^{5}$, Yan Sun ${ }^{3}$, Wujun Shi ${ }^{3}$, Zhe Zhang ${ }^{1}$, Ehrenfried Zschech ${ }^{4}$, Stefan C.B. Mannsfeld ${ }^{1}$, \\ Claudia Felser ${ }^{3} \&$ Xinliang Feng ${ }^{1}$
}

Metal-organic frameworks (MOFs) have so far been highlighted for their potential roles in catalysis, gas storage and separation. However, the realization of high electrical conductivity $\left(>10^{-3} \mathrm{~S} \mathrm{~cm}^{-1}\right)$ and magnetic ordering in MOFs will afford them new functions for spintronics, which remains relatively unexplored. Here, we demonstrate the synthesis of a twodimensional MOF by solvothermal methods using perthiolated coronene as a ligand and planar iron-bis(dithiolene) as linkages enabling a full $\pi$ - $d$ conjugation. This 2D MOF exhibits a high electrical conductivity of $\sim 10 \mathrm{~S} \mathrm{~cm}^{-1}$ at $300 \mathrm{~K}$, which decreases upon cooling, suggesting a typical semiconductor nature. Magnetization and ${ }^{57} \mathrm{Fe}$ Mössbauer experiments reveal the evolution of ferromagnetism within nanoscale magnetic clusters below $20 \mathrm{~K}$, thus evidencing exchange interactions between the intermediate spin $S=3 / 2$ iron(III) centers via the delocalized $\pi$ electrons. Our results illustrate that conjugated 2D MOFs have potential as ferromagnetic semiconductors for application in spintronics.

\footnotetext{
${ }^{1}$ Department of Chemistry and Food Chemistry \& Center for Advancing Electronics Dresden, Technische Universität Dresden, 01062 Dresden, Germany. ${ }^{2}$ Helmholtz-Zentrum Dresden-Rossendorf, Institute of Ion Beam Physics and Materials Research, Bautzner Landstr. 400, 01328 Dresden, Germany. ${ }^{3}$ Max Planck Institute for Chemical Physics of Solids, 01187 Dresden, Germany. ${ }^{4}$ Fraunhofer Institute for Ceramic Technologies and Systems (IKTS), 01109 Dresden, Germany. ${ }^{5}$ State Key Laboratory for Mechanical Behavior of Materials, Xi'an Jiaotong University, Xi'an 710049, China. Correspondence and requests for materials should be addressed to X.F. (email: xinliang.feng@tu-dresden.de)
} 
$\mathrm{T}$ he realization of high electrical conductivity and longrange magnetic ordering within a single material is highly appealing to meet the demands for data storage and processing 1,2 . Typically, ferromagnetic semiconductors are of considerable interest for spintronics (spin electronics) applications ${ }^{3-}$ 5 , where the electronic spins can be modulated by external electrical and magnetic fields to perform logic operations and act as memory. Currently, materials employed as ferromagnetic semiconductors generally comprise inorganic solid compounds like Heusler compounds and dilute magnetic semiconductors ${ }^{4,6-8}$ and organic/molecular film semiconductors ${ }^{9-16}$. In these inorganic solids, direct metal-metal bonds or indirect interactions among metal atoms mediated by small ligands (such as oxo atoms) enable the long-range electronic communication, which is essential to achieve the electrical conductivity and magnetic ordering ${ }^{4,6-8}$. Nevertheless, their entirely inorganic composition prevents a facile tunability of magnetic and conductive properties due to the lack of both ligand functionalization and structural diversity. In the case of molecular film semiconductors, their electronic and magnetic properties can be tuned to a much higher degree, due to the structural diversities of organic monomers and functional groups that can be employed. Notably, the molecular semiconductors such as vanadium-tetracyanoethylene ${ }^{11}$ and metal-phthalocyanine ${ }^{12-14}$ have exhibited strong magnetic coupling at high temperature, which are considered as an emerging class of spin transport media with long spin lifetime due to their carbon-based light-atom compositions. Nevertheless, the low mobility and complex transport properties in molecular semiconductors still hinder their practical applications for spintronics.

In contrast, metal-organic frameworks (MOFs) are hybrid porous materials based on crystalline coordination polymers consisting of metal atoms or clusters connected by organic ligands ${ }^{17,18}$. Their properties and functions can be tuned by varying abundant organic ligands, metal centers and framework geometries, thus enabling their potential in catalysis ${ }^{19}$, sensing ${ }^{20}$, gas storage $^{21,22}$ and separation ${ }^{23}$, etc. However, it is still challenging to realize magnetic MOF semiconductors. The conventionally reported three-dimensional (3D) MOFs showed extremely low bulk electrical conductivity $\left(10^{-12} \text { to } 10^{-8} \mathrm{~S} \mathrm{~cm}^{-1}\right)^{20,24}$ and weak magnetic coupling due to the large separation of metal centers by multi-atoms, insulating organic ligands, etc. Recent advances disclosed that the incorporation of conducting guests in MOFs could significantly enhance the electrical conductivity ${ }^{25,26}$. Moreover, immobilizing redox-active ligands with mixed-valences, such as 2,5-dihydroxybenzoquinone, into the backbones of iron(III) complexes could generate long-range charge delocalization and strong magnetic exchange ${ }^{27-29}$, leading to high conductivity $\left(\sim 10^{-4}\right.$ to $\left.\sim 0.2 \mathrm{~S} \mathrm{~cm}^{-1}\right)$ and high-temperature magnetic ordering (Curie temperature even could reach $105 \mathrm{~K}$ ). In addition, the design of conjugated 2D MOFs by employing planar organic ligands and square-planar metal-complex linkages inducing full delocalization of $\pi$-electrons has also led to improved electrical conductivity $\left(>10^{-3} \mathrm{~S} \mathrm{~cm}^{-1}\right)^{20,24}$. These reported $2 \mathrm{D}$ MOFs are based on benzene ${ }^{30-32}$ or triphenylene-derivatives ${ }^{33-36}$ with thiol ${ }^{30,31,35,36}$, hydroxyl ${ }^{33}$ or amino ${ }^{32,34}$ groups linked via $\mathrm{Ni}$ or $\mathrm{Cu}$ metal centers and have been integrated as electrode materials for applications in transistor ${ }^{37}$, electrocatalysis ${ }^{35,38,39}$, chemiresistive sensor $^{40}$ and energy storage ${ }^{41}$. Despite the magnetic behavior of these 2D MOFs remains unexplored, high delocalization of $\pi$-electrons in a $2 \mathrm{D}$ plane enables the interaction between charge carriers and localized spins to drive magnetic ordering $\mathrm{g}^{3,42}$, which makes conjugated $2 \mathrm{D}$ MOFs as intriguing candidates for ferromagnetic semiconductors ${ }^{42-44}$.

In this study, we demonstrate a 2D MOF (PTC-Fe) consisting of polycyclic aromatic hydrocarbon monomer, namely $1,2,3,4,5,6,7,8,9,10,11,12$-perthiolated coronene (PTC), as ligand connected by planar iron-bis(dithiolene) linkage enabling full $\pi-d$ conjugation in a $2 \mathrm{D}$ plane. Thus, such $2 \mathrm{D} \mathrm{MOF}$ is featured with hexagonal lattices and van der Waals layer-stacking structure. Four-probe van der Pauw electrical measurement revealed the room temperature conductivity value of $\sim 10 \mathrm{~S} \mathrm{~cm}^{-1}$ for bulk compressed pellet. A variable-temperature conductivity measurement displayed a non-linear increase of conductivity with temperature, indicating a typical semiconducting behavior. A density functional theory (DFT) calculation was carried out to estimate the band gap as $\sim 0.2 \mathrm{eV}$ for a monolayer MOF. A variable-temperature magnetic susceptibility measurement as well as ${ }^{57} \mathrm{Fe}$ Mössbauer spectra demonstrated that the PTC-Fe exhibited ferromagnetic ordering within nanoscale magnetic clusters at low temperatures (below $\sim 20 \mathrm{~K}$ ). Our work highlights conjugated 2D MOFs as a class of conductive materials exhibiting ferromagnetic and semiconducting features for potential spintronics application.

\section{Results}

Synthesis and structure. Black polycrystalline PTC-Fe 2D MOFs (Fig. 1a) were synthesized from reaction of $1,2,3,4,5,6,7,8,9,10$, 11,12-perthiolated coronene (PTC) ${ }^{45}$ with ammoniacal solutions of iron acetate $\left(\mathrm{Fe}(\mathrm{OAc})_{2}\right)$ in the mixture of deoxygenated water and dimethylformamide (DMF) heated at $120^{\circ} \mathrm{C}$ in a sealed vial for $48 \mathrm{~h}$. The solid product was collected and washed by deoxygenated DMF, dilute hydrochloric acid $(0.1 \mathrm{M})$, water and acetone and dried under vacuum at $100^{\circ} \mathrm{C}$. The attenuated total reflection IR (ATR-IR) spectra of PTC-Fe (Supplementary Fig. 1) displayed that the S-H signals at $2512 \mathrm{~cm}^{-1}$ disappeared in the PTC-Fe, suggesting that the thiol groups in monomer PTC were efficiently coordinated to $\mathrm{Fe}$ atoms. Thermogravimetric analysis (TGA) revealed that PTC-Fe decomposed above $300^{\circ} \mathrm{C}$, as evidenced by the pronounced weight losses above this temperature (Supplementary Fig. 2). The nitrogen sorption isotherms were measured at $77 \mathrm{~K}$ to evaluate the porosity of PTC-Fe (Supplementary Fig. 3), which showed gas uptake and release during the adsorption and desorption processes. The Brunauer-EmmettTeller specific surface area was calculated to be $210( \pm 5) \mathrm{m}^{2} / \mathrm{g}$ with the existence of counter ions of $\mathrm{NH}_{4}+$ in the pores.

Powder X-ray diffraction (PXRD) measurements with Co Ka irradiation $(\lambda=1.79 \AA)$ reveal a highly crystalline structure in PTC-Fe with prominent (100), (200) and (210) peaks at $2 \theta=8.8^{\circ}$, $17.6^{\circ}$, and $21.7^{\circ}$, respectively, as shown in Fig. 1b. We simulated several possible stacking arrangements for the layers of PTC-Fe by DFT calculation (Supplementary Fig. 4). The experimentally resolved pattern agrees well with the $\mathrm{AB}$ packing model with $25 \%$ shifting in $\mathrm{X}$ and $\mathrm{Y}$ directions between neighboring layers. Thus, the PXRD results combined with the theoretical simulation provide a structural model of PTC-Fe (Fig. 1b and enlarged image shown in Supplementary Fig. 4d), which exhibits a hexagonal pattern within the $a b$ planes and a $\mathrm{AB}$ layer stacking along the $c$ direction. The square planar, conjugated iron-bis(dithiolene) linkages extend the $\pi$-electron conjugation of coronene in a $2 \mathrm{D}$ plane. From the peak at $2 \theta=8.8^{\circ}$, we can infer a hexagonal unit cell with $a=b=\sim 11.7 \AA$. The peak at $2 \theta=26.0^{\circ}$, corresponding to the (001) reflection suggests an ordered stacking with an interlayer distance of $\sim 3.9 \AA$.

Transmission electron microscopy (TEM) studies provide further evidence of $2 \mathrm{D}$ long-range order and layer stacking in PTC-Fe. The TEM images show a non-porous morphology in PTC-Fe (Fig. 1c and Supplementary Fig. 5). In-plane periodicity is determined by selected area electron diffraction (SAED, inset in Fig. 1c), which presents strong polycrystalline diffraction rings. The ring size reveals an ordered network with $a=\sim 1.2 \mathrm{~nm}$ cell size. High resolution TEM (HRTEM) images clearly present 
a

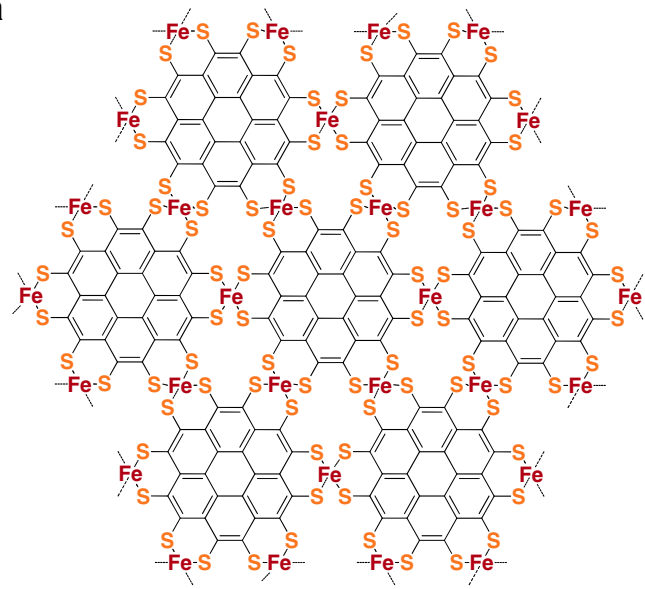

b

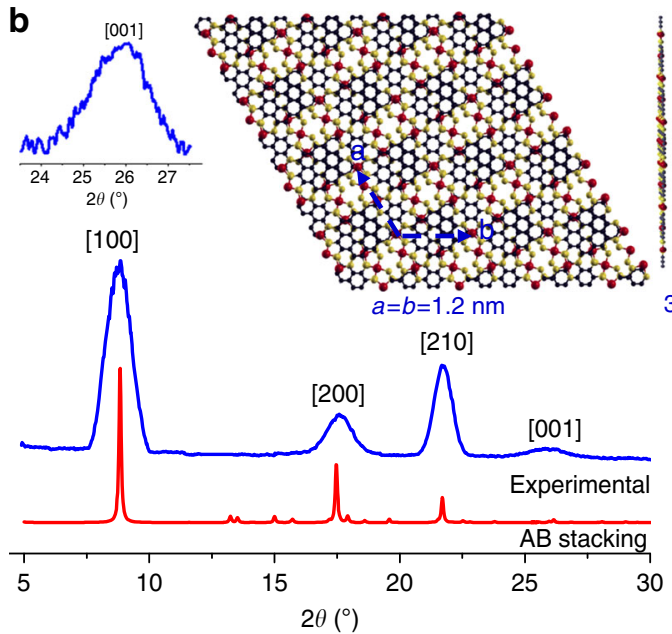

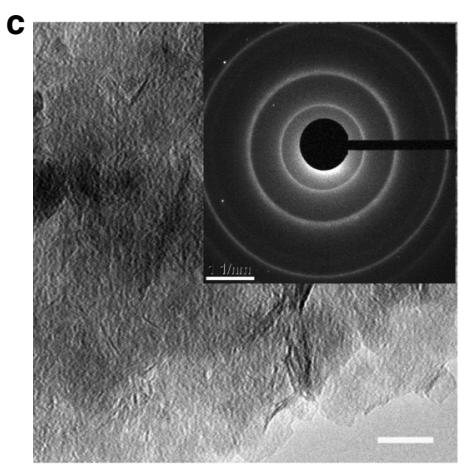
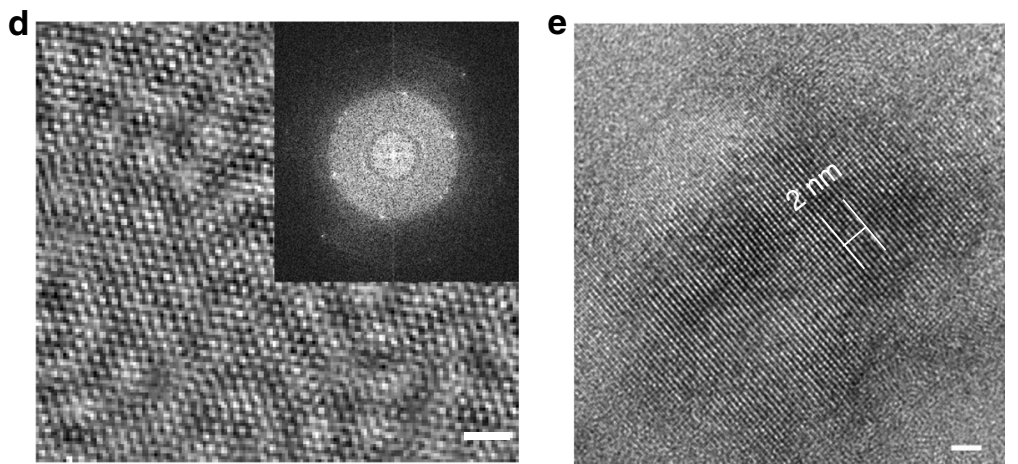

Fig. 1 Structural characterizations of PTC-Fe 2D MOF. a Schematic structure of PTC-Fe. b Experimental and simulated PXRD patterns. Insets: enlarged experimental PXRD peak corresponding to (001) reflection and the crystal structure simulation of $A B$ stacking model with $25 \%$ shifting in $X$ and $Y$ directions. c transmission electron microscopy (TEM) image. Scale bar $=50 \mathrm{~nm}$. Inset: Selected area electron diffraction (SAED) pattern. Scale bar=1 nm ${ }^{-1}$. d high-resolution in-plane TEM images. Scale bar $=2 \mathrm{~nm}$. Inset: fast Fourier transform (FFT) pattern from image (d). e high resolution TEM image from the side view. Scale bar $=2 \mathrm{~nm}$. The distance across five lattices is $2 \mathrm{~nm}$

crystalline domains within a dozen of nanometers (Fig. 1d and Supplementary Fig. 5). Fast Fourier transform (FFT) analysis of a crystalline domain in Fig. 1d further demonstrates a hexagonal structure with a lattice parameter $a=b=\sim 1.2 \mathrm{~nm}$, in excellent agreement with PXRD and DFT results. A cross view of PTC-Fe sample shows the layer-stacking structure with an interlayer distance of $\sim 4 \AA$ (Fig. 1e).

Compositional characterization. Next we investigated the composition of PTC-Fe. X-ray photoelectron spectroscopy (XPS) spectrum discloses the presence of Fe 2p, N 1 s, C 1 s, S 2 s, and S $2 \mathrm{p}$ core levels (Supplementary Fig. 6a). The $\mathrm{N}$ signal originates from the counter ions of $\mathrm{NH}_{4}+$ balancing the charged system. The high-resolution $\mathrm{Fe} 2 \mathrm{p}$ photoemission spectrum shows two sets of peaks (Supplementary Fig. 6b), with binding energies of 710.9 and $723.9 \mathrm{eV}$, which are assigned to the $2 \mathrm{p} 3 / 2$ and $2 \mathrm{p} 1 / 2$ levels, respectively, indicating the presence of Fe(III) species. The $\mathrm{S} 2 \mathrm{p}$ peak in the XPS spectrum occurs at a binding energy of $\sim 162 \mathrm{eV}$. Deconvolution of the S $2 \mathrm{p}$ signal generates highintensity dual peaks at 161.5 and $162.7 \mathrm{eV}$ derived from the $\mathrm{Fe}-\mathrm{S}$ units, evidencing efficient complexation between Fe ions and thiol groups (Supplementary Fig. 6c). Quantitative analysis of the Fe and $S$ signals provides a Fe:S ratio of $\sim 0.98: 4$, which is in consistence with the expected PTC-Fe model.

Synchrotron powder X-ray absorption spectroscopy (XAS) was employed to characterize the coordination geometry and iron valence in PTC-Fe (Fig. 2a and Supplementary Methods). As a reference, the known one-dimensional coordination polymer (TTB$\mathrm{Fe})^{46}$ comprising 1,2,4,5-tetrathiolbenzene and iron (III), as well as $\mathrm{Fe}$ foil, $\mathrm{FeO}$ and $\mathrm{Fe}_{2} \mathrm{O}_{3}$ inorganic solids were also investigated by XAS. Thus, the Fe K-edge X-ray absorption near edge structure (XANES) spectra of PTC-Fe reveal the same coordination geometry as the TTB-Fe. Figure $2 \mathrm{~b}$ shows the Fourier transform of the $\kappa$-weighted extended X-ray absorption fine structure (EXAFS) spectra of PTC-Fe, as well as $\mathrm{Fe}_{2} \mathrm{O}_{3}$ and TTB-Fe as contrast samples. The EXAFS spectra present a predominant peak in PTC$\mathrm{Fe}$, which is originated from the nearest-neighboring sulfur coordination shell around the Fe atoms. Based on this peak, Fe-S distance was calculated to be $\sim 2.23 \AA$. Besides this first-shell interaction, the second-shell atomic interactions were also observed, based on which distance of $\mathrm{Fe}-\mathrm{C}$ of $\sim 3.39 \AA$ was calculated (Supplementary Fig. 7). These bond lengths are very close to those of the contrast sample TTB-Fe, which are well consistent with the iron-bis(dithiolene) geometry. However, another contrast sample $\mathrm{Fe}_{2} \mathrm{O}_{3}$ exhibits two predominant peaks at $\sim 1.44 \AA$ and $\sim 2.57 \AA$, which arise from $\mathrm{Fe}-\mathrm{O}$ and $\mathrm{Fe}-\mathrm{Fe}$ bonds, respectively. Therefore, the XANES and EXAFS spectra of PTC-Fe and the contrast experiments provide strong proof on the formation of square planar iron-bis(dithiolene) complexes via the coordination of PTC and Fe ions. Moreover, no metal oxides such as $\mathrm{FeO}$ and $\mathrm{Fe}_{2} \mathrm{O}_{3}$ were detected in the PTC-Fe.

In order to further investigate the iron coordination geometry, local electronic structure, and magnetic properties, ${ }^{57} \mathrm{Fe}$ Mössbauer spectra were measured between 300 and $5 \mathrm{~K}$ (Fig. 2c, d, Supplementary Methods, Supplementary Fig. 8 and 

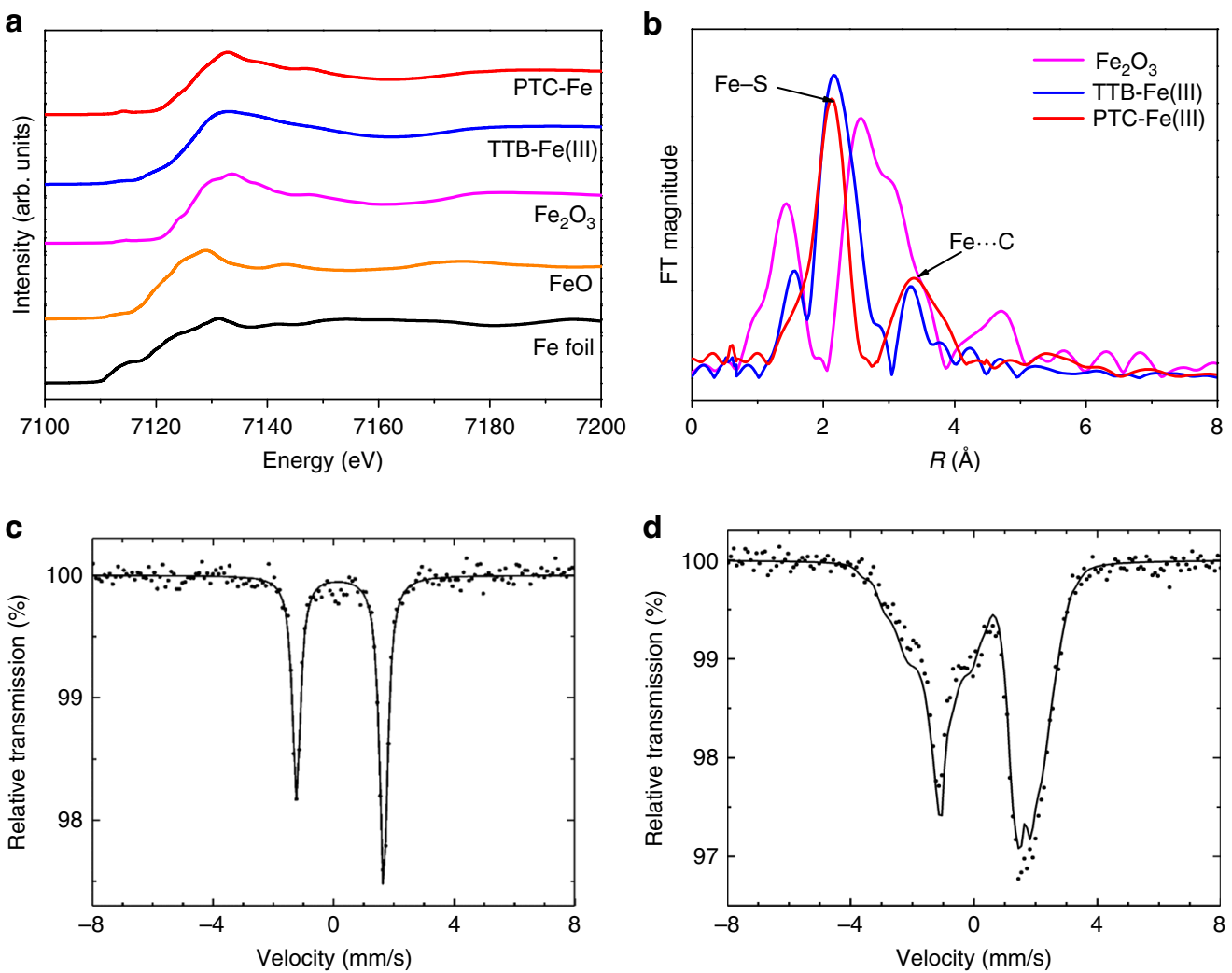

Fig. 2 Compositional characterizations of PTC-Fe. a Normalized XANES spectra at the Fe K-edge of PTC-Fe and its reference compounds, including Fe foil, $\mathrm{FeO}, \mathrm{Fe}_{2} \mathrm{O}_{3}$, and 1,2,4,5-tetrathiolbenzene-Fe(III) (TTB-Fe(III)). For the PTC-Fe, the energy of adsorption edge (EO) suggests that the valence state of the Fe ion in the PTC is $3+$. b Fourier transform of the EXAFS at Fe K-edge of PTC-Fe as well as $\mathrm{Fe}_{2} \mathrm{O}_{3}$ and TTB-Fe as the contrast samples. $\mathbf{c}$, $\mathbf{d}{ }^{57} \mathrm{Fe}$ Mössbauer spectra at $294 \mathrm{~K}$ and $5 \mathrm{~K}$, respectively. Dots and black line correspond to experimental data and calculated spectrum, respectively

Supplementary Table 1). Spectra obtained by cooling down from 300 to $10 \mathrm{~K}$ mainly feature a single quadrupole doublet. The isomer shift IS $=0.21 \mathrm{~mm} / \mathrm{s}$ and quadrupole splitting QS $=2.89$ $\mathrm{mm} / \mathrm{s}$ at $T=300 \mathrm{~K}$ are within the range of values typical for $\mathrm{Fe}$ (III) complexes with thiolate ligands and square planar coordination geometry 47,48 , which is in agreement with the anticipated Fe-bis(dithiolene) coordination in PTC-Fe. The intensity asymmetry of the two doublet components is a texture effect reflecting preferred orientation of crystallites in the sample. The $d$-orbital splitting pattern in square planar ligand environment is known to stabilize an intermediate spin $S=3 / 2$ state for $\mathrm{Fe}^{3+}$, and the large quadrupole splitting reflects the unequal electron population of the Fe $3 d$ orbitals split by the ligand field (valence contribution to the electric field gradient) ${ }^{27,49}$. The $\mathrm{S}=$ $3 / 2$ (intermediate spin) ground state of $\mathrm{Fe}^{3+}$ is a consequence of the square planar coordination geometry, which leads to a pronounced energetic stabilization of the $3 d z^{2}$ orbital with respect to the $d x^{2}-y^{2}$ orbital, and the energy of the $d z^{2}$ orbital is comparable to the energies of the $d x y, d x z$, and $d y z$ orbitals. Compared with the Fe(III), somewhat larger isomer shifts are expected for square planar $\mathrm{Fe}$ (II) complexes with an intermediate spin $S=1$. The temperature dependence of $I S$ reflects the usual second-order Doppler shift. A broadened quadrupole doublet (Supplementary Fig. 8) is observed at $10 \mathrm{~K}$; however, at $5 \mathrm{~K}$, the Mössbauer spectrum exhibits magnetic hyperfine splitting suggesting a magnetic ordering transition (Fig. 2d). Furthermore, Mössbauer spectra at low temperature also prove no iron oxides purities in the PTC-Fe.

Electronic structure of PTC-Fe. We measured solid-state UVVis spectrum of PTC-Fe (Supplementary Fig. 9). Importantly, the electronic absorption features of PTC-Fe extend well into the near-infrared (NIR) range. Such low-energy electronic excitations are common in highly conjugated organic/metal-organic and conducting polymers ${ }^{26}$. In order to estimate the electronic band structure of PTC-Fe, DFT calculations were performed (Fig. 3, Supplementary Methods and Supplementary Figs. 10-12). The density of states (DOS) describe the type and the number of states occupied at a certain energy and are essential for determining the carrier concentrations and energy distributions within a semiconductor $^{50}$. In this respect, we provide the DOS results for both the single layer (Fig. 3a, b, Supplementary Figs. 10, 11) and multilayer of the 2D MOF with $\mathrm{AB}$ stacking model (Fig. 3c, d, Supplementary Fig. 12). Spin up, spin down and mixed spin directions have been considered in the DOS calculation. The band structure simulation for the single layer indicates a band gap of $\sim 0.2 \mathrm{eV}$ (Fig. 3a, b), while that for the AB stacking system exhibits a metallic character (Fig. $3 \mathrm{c}, \mathrm{d}$ ). In addition, the single layer and $\mathrm{AB}$ stacking models present a band gap of $\sim 0.7 \mathrm{eV}$ and $\sim 1 \mathrm{eV}$ for the spin-up channel, respectively (Fig. $3 b, d$ ). However, for spin down channel, a semi-conducting feature remains for single layer while a metallic character is dominantly presented for $\mathrm{AB}$ stacking. Layer stacking is believed to narrow the band gap for most of the $2 \mathrm{D}$ materials ${ }^{51}$. In addition, due to the negatively charged structure in PTC-Fe, a compensating cation $\left(\mathrm{NH}_{4}{ }^{+}\right)$has been introduced into the cell to avoid electrostatic potential and energy diverge induced by net charge, which could also play a crucial role in narrowing the band gap. Around the Fermi level the dominating states are coming from Fe- $d$ and S- $p$ orbitals (Supplementary Fig. 10a and c). Analysis of the projected density of states (PDOS) near to the Fermi level show a hybridization of the $p$ orbitals of $\mathrm{S}$ and the $d y z$ - and $d x z$-orbitals of $\mathrm{Fe}$, suggesting that the $p$-orbitals of the system are delocalized (Supplementary 

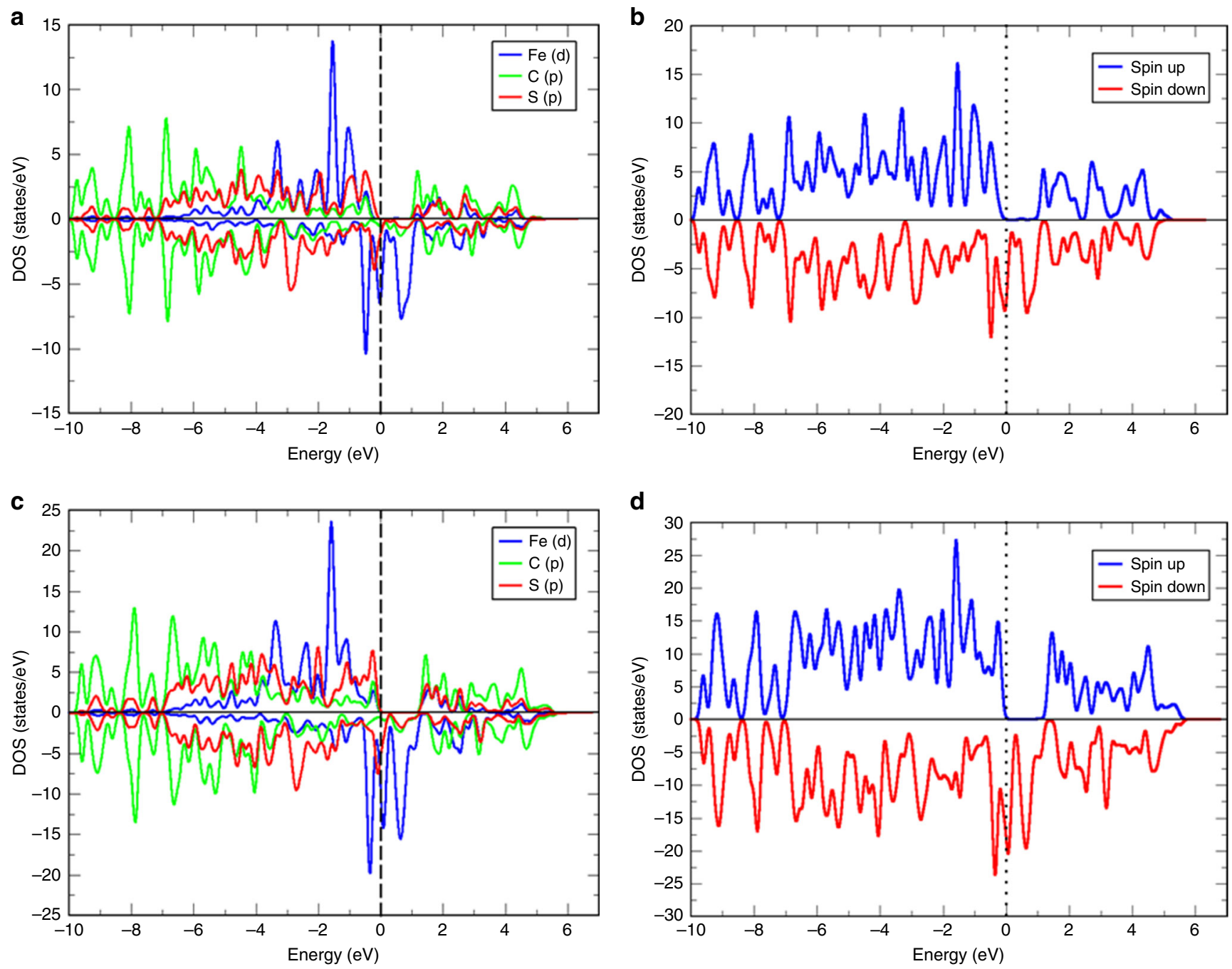

Fig. 3 Electronic band structure of PTC-Fe near the Fermi level. a Total density of states (DOS) for a single layer. b Spin up/Spin down for a single layer. c Total DOS for $A B$ stacking. d Spin up/Spin down for $A B$ stacking

Fig. $10 \mathrm{~b}$ and $\mathrm{d}$ ). The bulk materials are expected to be semimetallic in the $a b$ directions (due to the metal ions and the $\mathrm{S}$ atoms) and semiconducting in the $c$ direction due to weak van der Waals interactions.

DC conductivity measurements. The bulk electrical properties of PTC-Fe were determined from pelletized samples with the thickness of $\sim 0.3 \mathrm{~mm}$ obtained by pressing powders at $\sim 1 \mathrm{GPa}$ (Inset in Fig. $4 \mathrm{a}$ ). The $I-V$ curves were measured in the van der Pauw geometry and displayed Ohmic response between -1.0 and $+1.0 \mathrm{~V}$ (Supplementary Fig. 13), which gave an electrical conductivity of the pellets as high as $\sim 10 \mathrm{~S} \mathrm{~cm}^{-1}$ at $300 \mathrm{~K}$. This is among the highest values for the thus far reported intrinsically conducting MOF powders (Supplementary Table 2) 20,24,32.

The variable-temperature conductivity measurements showed a non-linear increase of conductivity from 20 to $320 \mathrm{~K}$ (Fig. 4a), indicating a typical semiconducting feature. The natural logarithm of conductivity $(\ln \sigma)$ plotted versus reciprocal temperature $(1 / T)$ is presented in Fig. $4 \mathrm{~b}$. The plot shows a linear region over a temperature range of $125-300 \mathrm{~K}$, consistent with a thermally activated transport dominant in this temperature range. The slope of the linear region in Fig. $4 \mathrm{~b}$ corresponds to the activation energy $\left(E_{a}\right)$, which can be extracted from the following Arrhenius law: $\sigma \sim \exp \left(E_{a} / k T\right)$, where $\sigma$ is the conductivity, $E_{a}$ is the activation energy, $k$ is the Boltzmann constant $\left(8.617 \times 10^{-5} \mathrm{eV} / \mathrm{K}\right)$ and $T$ is the temperature 31 . The value of $E_{a}$ was evaluated from the slope of $\ln \sigma$ versus $1 / T$ plot and was calculated as $0.2 \mathrm{eV}$. As shown in the inset of Fig. $4 \mathrm{~b}$, the plot of $\ln (\sigma)$ versus $T^{-1 / 4}$ over the temperature region $65-130 \mathrm{~K}$, which is well fitted to the Mott variable range hoping (Mott-VRH) model $^{52}$. We ascribe this hopping process to the grain boundaries between the crystallites dominating the temperature dependence of conductivity in the bulk polycrystalline pellets, giving rise to apparent semiconducting behavior ${ }^{32}$.

Magnetic properties of PTC-Fe. Iron-based MOFs [Fe(III)] can exhibit magnetic exchange interactions between neighboring iron atoms through the bridging ligands ${ }^{27,29}$. In order to probe the magnetic properties of the PTC-Fe, we measured the magnetization using a superconducting quantum interference devicevibrating sample magnetometer (SQUID-VSM) (Fig. 5).

Figure $5 \mathrm{a}$ and Supplementary Fig. 14a show the variation of the magnetization with applied magnetic field $(H)$ measured at different temperatures. While at $50 \mathrm{~K}$ the magnetization increases linearly with field, as expected for a paramagnetic system, a sigmoidal shape of $M(H)$ below $\sim 20 \mathrm{~K}$ signals the onset of 

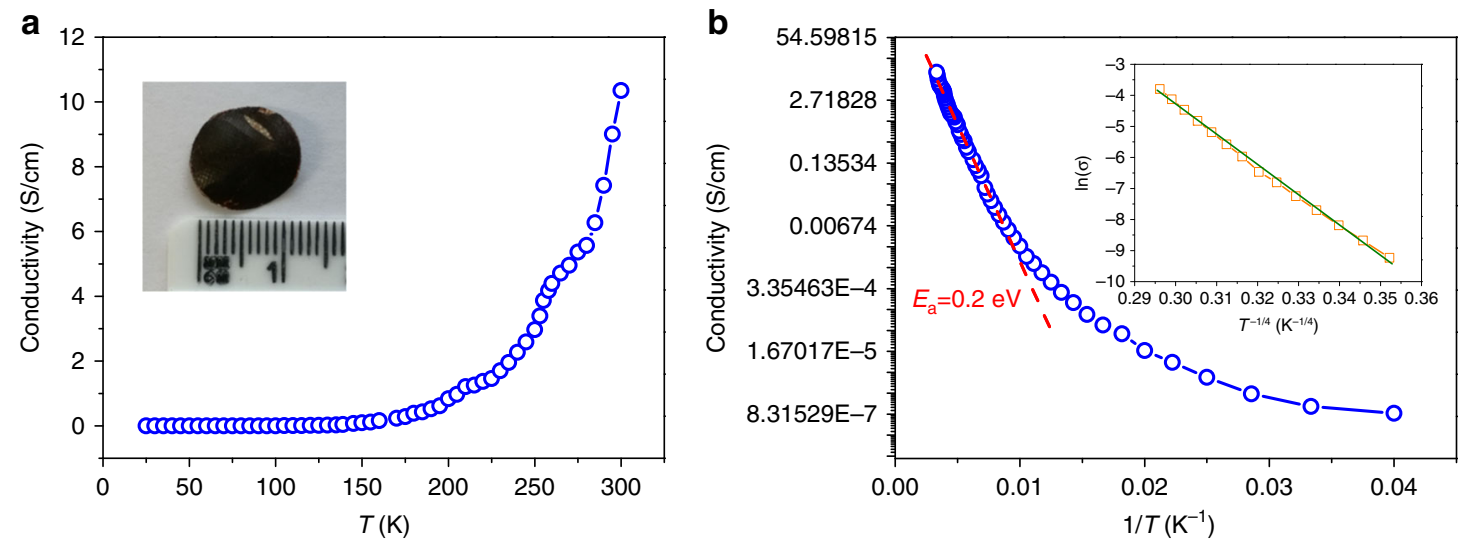

Fig. 4 Electrical conductivity of PTC-Fe 2D MOFs. a Electrical conductivity $(\sigma)$ as a functional of temperature ranging from $30 \mathrm{~K}$ to $300 \mathrm{~K}$. b Plot of In $\sigma$ versus the reciprocal of the temperature $(1 / T)$. Inset: Plot of $\ln (\sigma)$ versus $T^{-1 / 4}$ over the temperature region $65-130 \mathrm{~K}$
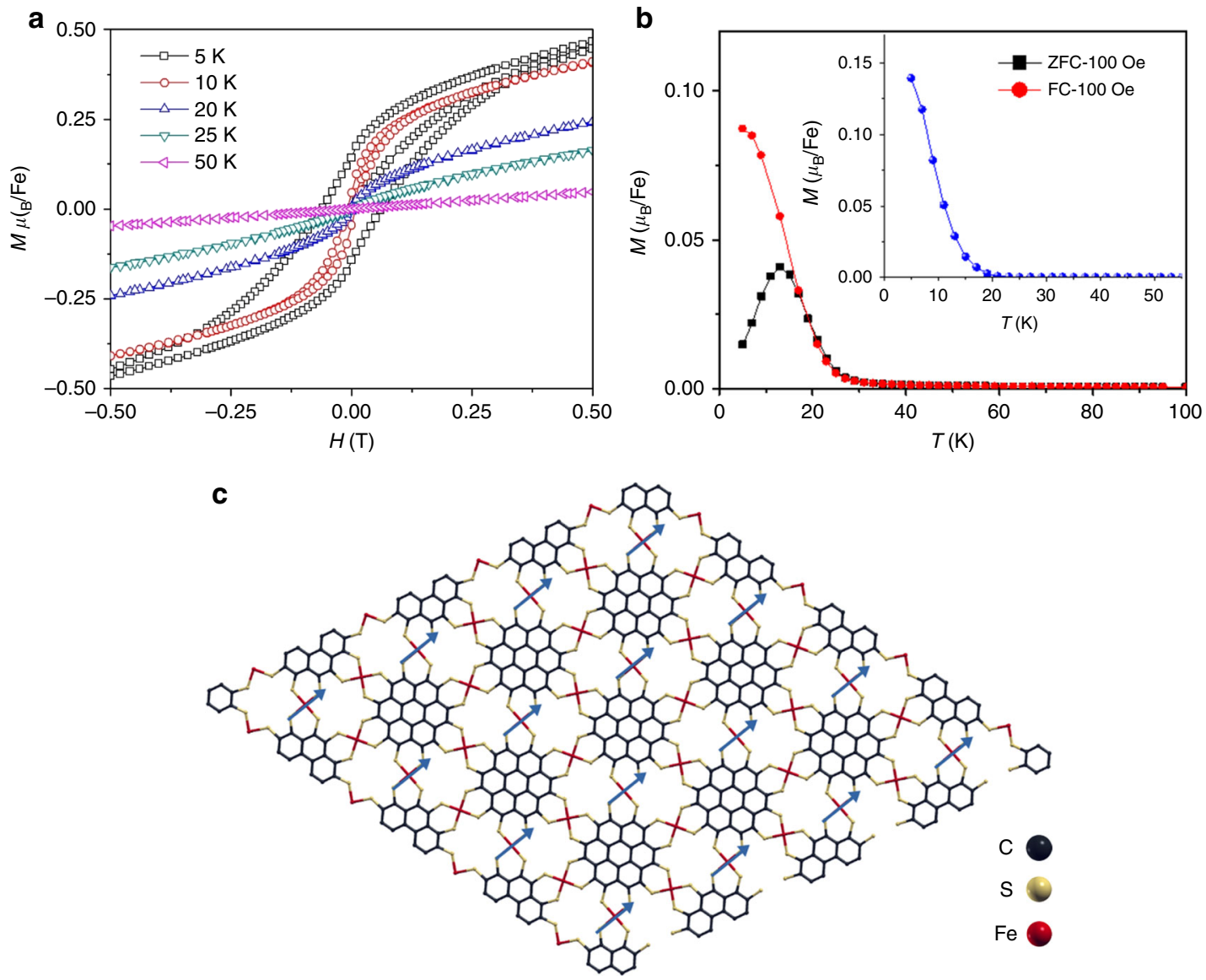

Fig. 5 Magnetic properties of PTC-Fe. a Magnetizations as functions of applied magnetic field (H) measured at different temperatures. b Magnetization of PTC-Fe as a function of temperature measured in a 100 Oe field under field-cooled (FC) and zero-field-cooled (ZFC) conditions. Inset: Temperature dependent remanent magnetization. c ferromagnetic ground state of PTC-Fe

ferromagnetism. For temperatures $T \leq 10 \mathrm{~K}$ a hysteresis is evident and the hysteresis width increases with decreasing temperature (coercive field $\sim 0.07 \mathrm{~T}$ at $5 \mathrm{~K}$ ). Furthermore, the $T$-dependent magnetization data (Fig. 5b) feature an increase in $M$ below $20 \mathrm{~K}$ and a cusp in the zero-field cooled (ZFC) magnetization near 15 $\mathrm{K}$. Below $15 \mathrm{~K}$ the FC and ZFC data diverge. These observations point to nanoscale ferromagnetism with an average blocking temperature of about $15 \mathrm{~K}^{53}$. The evolution of ferromagnetism below $\sim 20 \mathrm{~K}$ is in agreement with the variable-temperature magnetic remanence measurements (inset in Fig. 5b). These clues are in accordance with the Mössbauer spectra (Supplementary Fig. 8) where line broadening near $10 \mathrm{~K}$ signals the onset of spin freezing on the Mössbauer time scale $\left(\sim 10^{-8} \mathrm{~s}\right)$, while the broad hyperfine pattern at $5 \mathrm{~K}$ verifies that the majority of the sample is in a frozen magnetic state. However, the very broad hyperfine field distribution (Supplementary Fig. 8, bottom) suggests a wide distribution of relaxation times due to a variation in the size of the magnetic nano-domains. 


\section{Discussion}

To understand the origin of the relatively high-temperature $(<20$ $\mathrm{K})$ ferromagnetic semiconducting ground state in PTC-Fe, we investigated the magnetic properties of the organic ligand PTC as well as coronene as contrast samples by SQUID. The temperature dependent remanent magnetization plots reveal that the ligand PTC and the building core coronene contribute ignorable magnetic ordering even at $5 \mathrm{~K}$ while the coordination complexes comprising PTC and Fe behave ferromagnetism (Supplementary Fig. 15). We then evaluated the spin density distribution in ABstacking PTC-Fe by DFT calculation (Supplementary Fig. 16), which suggests that the $\mathrm{Fe}$ atoms provide the major spins compared with the $\mathrm{C}$ and $\mathrm{S}$ atoms, associated with localized $d$ electrons ${ }^{44}$. In this case, Fig. $5 \mathrm{c}$ shows the magnetic ground states of the PTC-Fe with unit-cell magnetization, in which the magnetic moments are predominantly localized on the iron atoms. Notably, the magnetic ground state of the system at $0 \mathrm{~K}$ will be ferromagnetic if the magnetic exchange energy is positive whereas anti-ferromagnetic if negative. Here, an exchange energy of $E_{\mathrm{ex}}=1.22 \mathrm{meV}\left(E_{\mathrm{ex}}=E_{\mathrm{AFM}}-E_{\mathrm{FM}}\right)$ has been obtained by DFT calculation, further revealing the intrinsic ferromagnetic character of this $2 \mathrm{D} \mathrm{MOF} 43,54$. The simulated Curie temperature of PTC-Fe is $\sim 16 \mathrm{~K}$ by employing Ising model (calculation seen in Supplementary Methods) ${ }^{54}$, which further supports the SQUID measurements on the magnetic ordering with a blocking temperature of $\sim 15 \mathrm{~K}$ (Fig. 5b).

With the preliminary determination of spin distribution, we attempt to further achieve an understanding how the magnetic coupling appears between the localized spins on Fe toms. Given the distance between the neighboring lateral Fe atoms from $\sim 0.54$ to $\sim 1.20 \mathrm{~nm}$ in PTC-Fe MOF (Fig. $1 \mathrm{~b}$ and Supplementary Fig. 7), direct exchange between the $d$ orbitals of lateral $\mathrm{Fe}$ is relatively weak, and thus cannot be responsible for the strong ferromagnetic coupling between the intralayer $\mathrm{Fe}$ atoms. In addition, the layer distance is $\sim 0.38 \mathrm{~nm}$ and the distance between the neighboring interlayer Fe atoms is $\sim 0.5 \mathrm{~nm}$, also leading to relatively weak direct exchange interaction. Thus, we propose that the magnetic coupling is induced by indirect exchange interaction ${ }^{14,27-29,43,44,55,56}$. Namely, the localized spin moments of the Fe atoms polarize the delocalized $p$ orbital through exchange interaction. That is because this layer-stacked PTC-Fe MOF comprises of fully conjugated planar structure, enabling strong hybridization between the $d / p$ orbitals of Fe, the coronene core, and the Fe-bis(dithiolene) nodes, which has a critical impact on the magnetic properties of the lattice. DOS plots also indicate that $d_{\mathrm{xz}} / d_{\mathrm{yz}}$ orbitals of Fe participate in the hybridization of $p$ orbitals (Supplementary Figs. 10 and 12), leading to strong exchange interaction between the localized $d$ orbitals and the $p$ orbitals, and hence ferromagnetic coupling. This indirect exchange through delocalized electrons is consistent with the Zener $d-p$ exchange ${ }^{42,57}$ or the Ruderman-Kittel-Kasuya-Yosida (RKKY) 58,59 exchange mechanism.

In summary, we have synthesized a coronene-based conjugated 2D MOF that exhibits record electrical conductivity of $\sim 10 \mathrm{~S} \mathrm{~cm}^{-1}$ for bulk compressed pellets at room temperature. The variabletemperature conductivity measurements revealed a typical semiconducting behavior for PTC-Fe. The variable-temperature magnetic susceptibility measurements indicated that PTC-Fe exhibits a ferromagnetic ground state at low temperature resulting from the unique hybridization between the $d / p$ orbitals of $\mathrm{Fe}$, the coronene core, and the Fe-bis(dithiolene) nodes. Our work indicates that endowing 2D MOFs with strongly delocalized $\pi$ systems can be an effective strategy to develop novel ferromagnetic MOF semiconductors. The possible contribution from the magnetic impurities to the ferromagnetic ordering has been excluded via the combined analysis of XPS, XANES, EXAFS, and
${ }^{57} \mathrm{Fe}$ Mössbauer spectra. It should be noticed that the conductive and magnetic behavior presented here did not rule out the impact of the defects possibly generated from grain boundaries, the crystallite tilting and the edges in the bulk materials ${ }^{60}$, due to the polycrystalline feature and structural complexity as a result of heterogeneity. In addition, the tuning of the defects generated from the uncoordinated vacancy sites was able to lead to longrange ferromagnetic coupling in MOFs, which have been significantly proved via theoretical and experimental approaches ${ }^{61}$. Our results hopefully encourage more physical studies on the ferromagnetic and semiconducting properties as well as spintronic applications, relying on the growth of MOF single-crystals or $2 \mathrm{D}$ crystalline films with tuned thickness and enlarged crystalline domain size by interfacial synthesis or chemical vapor deposition methods, which remains to be explored.

\section{Methods}

Materials. The ligand, 1,2,3,4,5,6,7,8,9,10,11,12-perthiolated coronene (PTC), was synthesized according to our previous reported protocols ${ }^{45}$. Starting from coronene, 1,2,3,4,5,6,7,8,9,10,11,12-dodecachlorocoronene was prepared by chlorination reaction. Nucleophilic replacement of all peripheral chlorosubstituents was achieved using lithium benzylthiolate at room temperature, which afforded $1,2,3,4,5,6,7,8,9,10,11,12$-dodecakis(benzylthio)coronene as a red powder in $62 \%$ yield. After reductive cleavage of the protective benzyl groups under Birch conditions using lithium in anhydrous liquid ammonia at $-78^{\circ} \mathrm{C}$, the dodecalithiocoronene-1,2,3,4,5,6,7,8,9,10,11,12-dodecathiolate was obtained. The subsequent direct treatment with aqueous hydrogen chloride and hydrogen peroxide afforded persulfurated coronene (PSC) in $61 \%$ isolated yield. The reduction of PSC by $\mathrm{NaBH}_{4}$ could afford PTC via the cleavage of S-S bonds.

Synthesis of PTC-Fe 2D MOFs. A degassed solution of $0.014 \mathrm{mmol}(10.0 \mathrm{mg})$ of PTC in $0.5 \mathrm{~mL}$ of DMF, $0.1 \mathrm{~mL}$ of aqueous ammonia $\left(\mathrm{NH}_{4} \mathrm{OH}, 6 \mathrm{M}\right)$, and a degassed solution of anhydrous $\mathrm{Fe}(\mathrm{OAc})_{2}(7.5 \mathrm{mg}, 0.043 \mathrm{mmol})$ in $0.5 \mathrm{~mL}$ of water were transferred into a $10 \mathrm{ml}$ glass vial and sonicated for $5 \mathrm{~min}$ at $0{ }^{\circ} \mathrm{C}$. Then, the vial was sealed and heated at $120^{\circ} \mathrm{C}$ in an oven for $48 \mathrm{~h}$, followed by natural cooling to room temperature. The solid product was afterwards collected and washed three times by deoxygenated DMF, dilute $\mathrm{HCl}(0.1 \mathrm{M})$, water and acetone. After dried at $100{ }^{\circ} \mathrm{C}$ under vacuum for $24 \mathrm{~h}, \sim 12 \mathrm{mg}$ of black crystals was obtained and stored in Ar. Anal. Calcd. for $\left[\mathrm{Fe}_{3} \mathrm{C}_{24} \mathrm{~S}_{12}\right]^{3-} \bullet\left(\mathrm{NH}_{4}{ }^{+}\right)_{3}: \mathrm{C}, 32.19 ; \mathrm{S}, 42.92 ; \mathrm{N}$, 4.69; $\mathrm{H}, 1.34$. Found: $\mathrm{C}, 32.41 ; \mathrm{S}, 43.12 ; \mathrm{N}, 4.45 ; \mathrm{H}, 1.68$, in which the $\mathrm{NH}_{4}{ }^{+}$are counter ions to balance the charges in the system.

Conductivity measurements. The solid sample was finely ground and pressed between two Mylar tapes (sample thickness is about $300 \mu \mathrm{m}$ and the diameter is around $1.2 \mathrm{~cm}$ ) at $\sim 1 \mathrm{GPa}$. The $\mathrm{Cu}$ wires were contacted on the pellet surface by carbon paint in a glove box. Thus, a four-point contact was placed at the circumference to define a square. The $I-V$ curves of the pellets were measured in van der Pauw geometry under vacuum at varied temperatures (from $20 \mathrm{~K}$ to $320 \mathrm{~K}$ ) using a commercial Lakeshore Hall System.

Magnetic studies. Magnetometry was performed using a SQUID-VSM (Quantum Design). Temperature dependence of the magnetization of the PTC-Fe 2D MOF powder sample was measured in zero-field cooling and field cooling sequence with applied magnetic field of $100 \mathrm{Oe}$. The magnetic field dependence of magnetization was measured at different temperatures, i.e., 5, 10, 20, 25, and $50 \mathrm{~K}$. Remanent magnetization was measured as a function of temperature in a zero magnetic field after the sample was cooled down from $350 \mathrm{~K}$ to $5 \mathrm{~K}$ in a magnetic field of $1000 \mathrm{Oe}$

Data availability. The data that support the findings of this study are available from the corresponding author on reasonable request.

Received: 20 December 2017 Accepted: 4 June 2018 Published online: 06 July 2018

\section{References}

1. Dechambenoit, P. \& Long, J. R. Microporous magnets. Chem. Soc. Rev. 40, 3249-3265 (2011).

2. Awschalom, D. D. \& Flatté, M. E. Challenges for semiconductor spintronics. Nat. Phys. 3, 153-159 (2007).

3. Dietl, T. A ten-year perspective on dilute magnetic semiconductors and oxides. Nat. Mater. 9, 965-974 (2010). 
4. Dietl, T. \& Ohno, H. Dilute ferromagnetic semiconductors: physics and spintronic structures. Rev. Mod. Phys. 86, 187-251 (2014).

5. Dietl, T. Ferromagnetic semiconductors. Semicond. Sci. Technol. 17, 377-392 (2002).

6. Xu, Y., Awschalom, D. D. \& Nitta, J. Handbook of Spintronics. Vol. 9, 335-364 (Springer, Netherlands, 2016).

7. Chadov, S. et al. Tunable multifunctional topological insulators in ternary Heusler compounds. Nat. Mater. 9, 541-545 (2010).

8. Fedorych, O. M. et al. Magnetic order in semiconducting, ferromagnetic $\mathrm{Ga}_{1-\mathrm{x}} \mathrm{Mn}_{\mathrm{x}} \mathrm{As}$. Semicond. Sci. Technol. 19, S492 (2004).

9. Xiong, Z. H., Wu, D., Vardeny, Z. V. \& Shi, J. Giant magnetoresistance in organic spin-valves. Nature 427, 821-824 (2004).

10. Dediu, V. A., Hueso, L. E., Bergenti, I. \& Taliani, C. Spin routes in organic semiconductors. Nat. Mater. 8, 707-716 (2009).

11. Yoo, J. W. et al. Spin injection/detection using an organic-based magnetic semiconductor. Nat. Mater. 9, 638-642 (2010).

12. Warner, M. et al. Potential for spin-based information processing in a thinfilm molecular semiconductor. Nature 503, 504-509 (2013)

13. Serri, M. et al. High-temperature antiferromagnetism in molecular semiconductor thin films and nanostructures. Nat. Commun. 5, 3079 (2014).

14. Dhara, B. et al. Possible room-temperature ferromagnetism in self-assembled ensembles of paramagnetic and diamagnetic molecular semiconductors. J. Phys. Chem. Lett. 7, 4988-4995 (2016).

15. Tsurumi, J. et al. Coexistence of ultra-long spin relaxation time and coherent charge transport in organic single-crystal semiconductors. Nat. Phys. 13, 994-998 (2017).

16. Odenthal, P. et al. Spin-polarized exciton quantum beating in hybrid organic-inorganic perovskites. Nat. Phys. 13, 894-899 (2017).

17. Li, H., Eddaoudi, M., O'Keeffe, M. \& Yaghi, O. M. Design and synthesis of an exceptionally stable and highly porous metal-organic framework. Nature 402, 276-279 (1999).

18. Furukawa, H., Cordova, K. E., O’Keeffe, M. \& Yaghi, O. M. The chemistry and applications of metal-organic frameworks. Science 341, 1230444 (2013).

19. Zhang, T. \& Lin, W. Metal-organic frameworks for artificial photosynthesis and photocatalysis. Chem. Soc. Rev. 43, 5982-5993 (2014).

20. Stassen, I. et al. An updated roadmap for the integration of metal-organic frameworks with electronic devices and chemical sensors. Chem. Soc. Rev. 46, 3185-3241 (2017).

21. Eddaoudi, M. et al. Systematic design of pore size and functionality in isoreticular MOFs and their application in methane storage. Science 295, 469-472 (2002).

22. Murray, L. J., Dinca, M. \& Long, J. R. Hydrogen storage in metal-organic frameworks. Chem. Soc. Rev. 38, 1294-1314 (2009).

23. Li, J.-R., Sculley, J. \& Zhou, H.-C. Metal-organic frameworks for separations. Chem. Rev. 112, 869-932 (2012).

24. Sun, L., Campbell, M. G. \& Dincă, M. Electrically conductive porous metalorganic frameworks. Angew. Chem. Int. Ed. 55, 3566-3579 (2016).

25. Talin, A. A. et al. Tunable electrical conductivity in metal-organic framework thin-film devices. Science 343, 66-69 (2014).

26. Dhara, B. et al. Increase in electrical conductivity of MOF to billion-fold upon filling the nanochannels with conducting polymer. J. Phys. Chem. Lett. 7, 2945-2950 (2016).

27. Darago, L. E. et al. Electronic conductivity, ferrimagnetic ordering, and reductive insertion mediated by organic mixed-valence in a ferric semiquinoid metal-organic framework. J. Am. Chem. Soc. 137, 15703-15711 (2015).

28. Jeon, I.-R., Negru, B., Van Duyne, R. P. \& Harris, T. D. A 2D semiquinone radical-containing microporous magnet with solvent-induced switching from $T_{c}=26$ to 80 K. J. Am. Chem. Soc. 137, 15699-15702 (2016).

29. DeGayner, J. A. et al. $2 \mathrm{D}$ conductive iron-quinoid magnets ordering up to $T_{c}=105 \mathrm{~K}$ via heterogenous redox chemistry. J. Am. Chem. Soc. 139, 4175-4184 (2017).

30. Kambe, T. et al. $\pi$-Conjugated nickel bis(dithiolene) complex nanosheet. $J$. Am. Chem. Soc. 135, 2462-2465 (2013).

31. Huang, X. et al. A two-dimensional $\pi$ - $d$ conjugated coordination polymer with extremely high electrical conductivity and ambipolar transport behaviour. Nat. Commun. 6, 7408 (2015).

32. Dou, J.-H. et al. Signature of metallic behavior in the metal-organic frameworks $\mathrm{M}_{3}$ (hexaiminobenzene) ${ }_{2}(\mathrm{M}=\mathrm{Ni}, \mathrm{Cu})$. J. Am. Chem. Soc. 139, 13608-13611 (2017).

33. Hmadeh, M. et al. New porous crystals of extended metal-catecholates. Chem. Mater. 24, 3511-3513 (2012)

34. Sheberla, D. et al. High electrical conductivity in $\mathrm{Ni}_{3}(2,3,6,7,10,11$ hexaiminotriphenylene $)_{2}$, a semiconducting metal-organic graphene analogue. J. Am. Chem. Soc. 136, 8859-8862 (2014).

35. Dong, R. et al. Large-area, free-standing, two-dimensional supramolecular polymer single-layer sheets for highly efficient electrocatalytic hydrogen evolution. Angew. Chem. Int. Ed. 54, 12058-12063 (2015).
36. Clough, A. J. et al. Metallic conductivity in a two-dimensional cobalt dithiolene metal-organic framework. J. Am. Chem. Soc. 139, 10863-10867 (2017).

37. Wu, G. et al. Porous field-effect transistors based on a semiconductive metalorganic framework. J. Am. Chem. Soc. 139, 1360-1363 (2017).

38. Miner, E. M. et al. Electrochemical oxygen reduction catalysed by $\mathrm{Ni}_{3}$ (hexaiminotriphenylene) ${ }_{2}$. Nat. Commun. 7, 10942 (2016).

39. Dong, R. et al. Immobilizing molecular metal dithiolene-diamine complexes on $2 \mathrm{D}$ metal-organic frameworks for electrocatalytic $\mathrm{H} 2$ production. Chem. Eur. J. 23, 2255-2260 (2017).

40. Campbell, M. G. et al. $\mathrm{Cu}_{3}$ (hexaiminotriphenylene) $)_{2}$ : an electrically conductive 2D metal-organic framework for chemiresistive sensing. Angew. Chem. Int. Ed. 54, 4349-4352 (2015).

41. Sheberla, D. et al. Conductive MOF electrodes for stable supercapacitors with high areal capacitance. Nat. Mater. 16, 220-224 (2016).

42. Dietl, T. et al. Zener model description of ferromagnetism in zinc-blende magnetic semiconductors. Science 287, 1019-1022 (2000).

43. $\mathrm{Li}, \mathrm{W}$. et al. High temperature ferromagnetism in $\pi$-conjugated twodimensional metal-organic frameworks. Chem. Sci. 8, 2859-2867 (2017).

44. Chakravarty, C., Bikash Mandal, B. \& Sarkar, P. Coronene-based metalorganic framework: a theoretical exploration. Phys. Chem. Chem. Phys. 18, 25277-25283 (2016).

45. Dong, R. et al. Persulfurated coronene: a new generation of "sulflower". J. Am. Chem. Soc. 139, 2168-2171 (2017).

46. Dirk, C. W., Cox, S. D., Wellman, D. E. \& Wudl, F. Isolation and purification of benzene-1,2,4,5-tetrathiol. J. Org. Chem. 50, 2395-2397 (1985).

47. Dirk, C. W. et al. Metal poly(benzodithiolenes). Macromolecules 19, 266-269 (1986).

48. Sellmann, D., Geck, M. \& Moll, M. Transition-metal complexes with sulfur ligands. 62. Hydrogen evolution upon reaction of protons with sulfurcoordinated iron(II) complexes. Investigation of the proton, hydrogen and hydride interactions with iron 1,2-benzenedithiolate complexes. J. Am. Chem. Soc. 113, 5259-5264 (1991).

49. Roy, N. et al. Molecular and electronic structure of the square planar bis(oamidobenzenethiolato)iron(III) anion and its bis(o-quinoxalinedithiolato)iron (III) analogue. Inorg. Chem. 47, 10911-10920 (2008).

50. Kresse, G. \& Furthmüller, J. Efficiency of $a b$-initio total energy calculations for metals and semiconductors using a plane-wave basis set. Comput. Mater. Sci. 6, 15-50 (1996).

51. Kuc, A. \& Heine, T. The electronic structure calculations of two-dimensional transition-metal dichalcogenides in the presence of external electric and magnetic fields. Chem. Soc. Rev. 44, 2603-2614 (2015).

52. Wang, Y. et al. Ferromagnetism and crossover of positive magnetoresistance to negative magnetoresistance in $\mathrm{Na}$-doped $\mathrm{ZnO}$. Chem. Mater. 27, 1285-1291 (2015).

53. Friedländer, S. et al. Linear chains of magnetic ions stacked with variable distance: ferromagnetic ordering with a Curie temperature above $20 \mathrm{~K}$. Angew. Chem. Int. Ed. 55, 12683-12687 (2016).

54. Zhou, J. \& Sun, Q. Magnetism of phthalocyanine-based organometallic single porous sheet. J. Am. Chem. Soc. 133, 15113-15119 (2011).

55. Lannes, A. et al. Room temperature magnetic switchability assisted by hysteretic valence tautomerism in a layered two-dimensional manganeseradical coordination framework. J. Am. Chem. Soc. 138, 16493-16501 (2016).

56. Espallargas, G. M. \& Coronado, E. Magnetic functionalities in MOFs: from the framework to the pore. Chem. Soc. Rev. 47, 533-557 (2018).

57. Zener, C. Interaction between the $d$ shells in the transition metals. Phys. Rev. 81, 440-444 (1951).

58. Coey, J. M. D., Venkatesan, M. \& Fitzgerald, C. B. Donor impurity band exchange in dilute ferromagnetic oxides. Nat. Mater. 4, 173-179 (2005). 92005 .

59. Girovsky, J. et al. Long-range ferrimagnetic order in a two-dimensional supramolecular Kondo lattice. Nat. Commun. 8, 15388 (2017).

60. Fang, Z., Bueken, B., De Vos, D. E. \& Fischer, R. A. Defect-engineered metalorganic frameworks. Angew. Chem. Int. Ed. 54, 7234-7254 (2015).

61. Shen, L. Origin of long-range ferromagnetic ordering in metal-organic frameworks with antiferromagnetic dimeric-Cu(II) building units. J. Am. Chem. Soc. 134, 17286-17290 (2012).

\section{Acknowledgements}

This work was financially supported by the ERC Grant on 2DMATER, EU Graphene Flagship, SPP 1928 (COORNET) and the German Science Council. We acknowledge the cfaed (Center for Advancing Electronics Dresden). We also thank Beamline BL14W1 at the Shanghai Synchrotron Radiation Facility (SSRF) for providing the beamtimes to carry out the XAS measurements. We acknowledge Dresden Center for Nanoanalysis (DCN) at TUD and Dr. Petr Formanek (Leibniz Institute for Polymer Research, IPF, Dresden) for the use of facilities, and we like to appreciate Prof. Stuart Parkin, Dr. Binghai Yan, Dr. Reinhard Berger and Mr. Chi Xu for the helpful discussion. 


\section{Author contributions}

X.L.F. and R.H.D. conceived and designed the experiments. R.H.D. synthesized the 2D MOFs, and conducted structure, composition and property characterizations. R.H.D. and M.C.W. synthesized the PTC precursors. Z.T.Z., R.H.D. and S.Q.Z. contributed to the magnetic measurements and analysis. Z.T.Z., S.Q.Z., R.H.D., Z.Z. and S.M. contributed to the conductivity measurements and analysis. D.T., Y.S., W.S. and C.F. performed the theoretical simulation of the 2D MOFs. P.A. and C.F. contributed to the Mössbauer spectroscopy measurements. Z.Q.L., R.H.D. and E.Z. carried out the HRTEM measurements. F.L. performed the X-adsorption spectroscopy experiments. R.H.D. and X.L. F. co-wrote the paper. All authors discussed the results and commented on the manuscript.

\section{Additional information}

Supplementary Information accompanies this paper at https://doi.org/10.1038/s41467018-05141-4.

Competing interests: The authors declare no competing interests.

Reprints and permission information is available online at http://npg.nature.com/ reprintsandpermissions/
Publisher's note: Springer Nature remains neutral with regard to jurisdictional claims in published maps and institutional affiliations.

\section{(c) (i)}

Open Access This article is licensed under a Creative Commons Attribution 4.0 International License, which permits use, sharing, adaptation, distribution and reproduction in any medium or format, as long as you give appropriate credit to the original author(s) and the source, provide a link to the Creative Commons license, and indicate if changes were made. The images or other third party material in this article are included in the article's Creative Commons license, unless indicated otherwise in a credit line to the material. If material is not included in the article's Creative Commons license and your intended use is not permitted by statutory regulation or exceeds the permitted use, you will need to obtain permission directly from the copyright holder. To view a copy of this license, visit http://creativecommons.org/ licenses/by/4.0/.

(C) The Author(s) 2018 